\section{Lipid droplets characterization in adipocyte differentiated 3T3-L1 cells: size and optical density distribution}

\author{
V. Rizzatti, ${ }^{1}$ F. Boschi, ${ }^{2}$ M. Pedrotti, \\ E. Zoico, ${ }^{1}$ A. Sbarbati, ${ }^{2}$ M. Zamboni ${ }^{1}$ \\ 'Department of Medicine, Geriatric \\ Section, University of Verona \\ 2Department of Neurological, \\ Neuropsychological, Morphological and \\ Motor Sciences, University of Verona, \\ Italy
}

\begin{abstract}
The 3T3-L1 cell line, derived from 3T3 cells, is widely used in biological research on adipose tissue. 3T3-L1 cells have a fibroblast-like morphology, but, under appropriate conditions, they differentiate into an adipocyte-like phenotype. During the differentiation process, 3T3-L1 cells increase the synthesis of triglycerides and acquire the behavior of adipose cells. In particular, triglycerides accumulate in lipid droplets (LDs) embedded in the cytoplasm. The number and the size distribution of the LDs is often correlated with obesity and many other pathologies linked with fat accumulation. The integrated optical density (IOD) of the LDs is related with the amount of triglycerides in the droplets. The aim of this study is the attempt to characterize the size distribution and the IOD of the LDs in 3T3-L1 differentiated cells. The cells were differentiated into adipocytes for 5 days with a standard procedure, stained with 0il Red 0 and observed with an optical microscope. The diameter, area, optical density of the LDs were measured. We found an asymmetry of the kerorganelles that regulate the storage and hydrolysis of neutral lipids, including triglycerides and cholesterol esters. ${ }^{8}$

The 0il red 0 staining was used in many different work to quantify the adipose conversion and triglycerides ${ }^{9}$ and loss of lipids in decellularized tissue. ${ }^{10}$ The integrated optical density (IOD) of the LDs was used to quantify the lipid accumulation in muscle fibers which was found significantly increased in obesity and reduced with weight loss. ${ }^{11}$ Moreover, Ramirez-Zacarias and co-workers found that, staining the adipocyte with 0 il red 0 , the IOD increases linearly with the triglycerides content in 3T3 adipocytes. ${ }^{9}$ LDs are dynamic and heterogeneous in size, location, and protein content. In literature it has been described the cellular role, processes of biogenesis, mobilization of fat and role of proteins associated with $\mathrm{LDs}^{12}$ but no one has comprehensively described the important aspect of their heterogeneity. The aim of this work was to quantify the heterogeneity in size and optical density of intracellular LDs in 3T3-L1 cells. This represents the first step in order to analyze fat accumulation in cells and the changes in pathological disorders.
\end{abstract} nel density distribution of the maximum Feret's diameter of the LDs with a tail due to very large LDs. More information regarding the birth of the LDs could help in finding the best mathematical model in order to analyze fat accumulation in adipocytes.

\section{Introduction}

The role of fat accumulation has emerged as a fundamental target of medical studies because it is responsible of obesity, a widespread disease in affluent societies. Obesity is a major cause of preventable death in the world. ${ }^{1-3}$ In particular, excessive triglycerides accumulation in humans can lead to cardiovascular dysfunction, diabetes mellitus type 2, hypertension, hypercholesterolemia, metabolic syndromes, hypertriglyceridemia, disorders of osteo-articular system, stroke, sleep apnea syndrome and some types of cancer. ${ }^{4,5}$ In recent years, in order to understand the molecular regulation of human obesity, immortalized murine cell lines such as 3T3-L1 cells and F442A were studied. ${ }^{6}$ 3T3-L1 is a cell line derived from 3T3 cells. 3T3-L1 cells have a fibroblast-like morphology, but, under appropriate conditions, they differentiate into an adipocyte-like phenotype The differentiation process of 3T3-L1 cells in adipocyte-like cells is based on the increased synthesis and accumulation of triglycerides, for this reason the 3T3L1 cells are extensively used to study adipogenesis. ${ }^{7}$ Triglycerides accumulate in lipid droplets (LDs) in the cytoplasm. LDs are intracellular

\section{Materials and Methods}

\section{Cell line and cell culture}

The 3T3-L1 preadipocytes (ECACC, SigmaAldrich, St. Louis, M0, USA) were cultured in $250 \mathrm{~mL}$ polystyrene sterilized flasks equipped with $0.2 \mu \mathrm{m}$ vented plug seal cap by BD (Becton Dickinson, Franklin Lakes, NJ, USA), with DMEM/GlutaMAX, supplemented with $10 \%$ of FBS, and 1\% of Antibiotic Antimycotic Solution, to reach a concentration of $2.5-3.0 \times 10^{6}$ cells (confluence 85-90\%). Then, 3T3-L1 cells were detached from flask with trypsin-EDTA and further eluted to be seeded in 4-wells BD Bio Coat $^{\mathrm{TM}}$ CultureSlides. Two days after confluence in wells, cells were washed three times
Correspondence: Federico Boschi, Department of Neurological, Neuropsychological, Morphological and Motor Sciences, University of Verona, strada Le Grazie 8, 37134 Verona, Italy.

E-mail: federico.boschi@univr.it

Key words: Lipid droplet, 3T3-L1, adipocyte, fat, triglyceride accumulation, integrated optical density.

Acknowledgments: the authors would like to thanks Dr. Lucia Calciano for her useful discussions in the statistical field.

Contributions: VR, data acquisition, analysis and interpretation; FB, concept, analysis and interpretation of data, draft of the article; MP, EZ, data acquisition; AS, MZ, revision of article for impor tant intellectual content.

Conflict of interests: the authors declare no conflict of interests.

Received for publication: 27 May 2013. Accepted for publication: 27 June 2013.

This work is licensed under a Creative Commons Attribution NonCommercial 3.0 License (CC BYNC 3.0).

(C) Copyright V. Rizzatti et al., 2013

Licensee PAGEPress, Italy

European Journal of Histochemistry 2013; 57:e24 doi:10.4081/ejh.2013.e24

with PBS and induced to differentiation with DMEM/F12 containing 10\% FBS, 1\% Antibiotic Antimycotic Solution, $0.2 \mathrm{mM}$ IBMX, $10 \mu \mathrm{M}$ rosiglitazone, $1 \mu \mathrm{M}$ dexamethasone, $10 \mu \mathrm{g} / \mathrm{mL}$ insulin for three days. After $72 \mathrm{~h}$ post-induction, cells underwent PBS washing (three times), and medium was replaced with an adipocyte maintaining medium (AMM), composed of DMEM/F12 enriched with 10\% FBS, 1\% Antibiotic Antimycotic Solution, $10 \mu \mathrm{g} / \mathrm{mL}$ insulin, in which cells were cultured for 2 days. 3T3-L1 cells were incubated at $37^{\circ} \mathrm{C}, 5 \% \mathrm{CO}_{2}$ in each culture step.

\section{Oil Red O staining}

After washing with PBS 0.1M pH 7.4, cell cultures were fixed for 20 min with $4 \%$ formalin in PBS 0.05M. Cells were washed with sterile double distilled water and subsequently with $60 \%$ isopropanol for $2 \mathrm{~min}$ and stained with a filtered $0.35 \%$ Oil Red 0 solution in $60 \%$ isopropanol for $10 \mathrm{~min}$ at room temperature. Then, cells were washed with sterile double distilled water and stained with Mayer's Hematoxylin Bio-Optica ready to use solution for $1 \mathrm{~min}$ at room temperature and washed again with sterile double distilled water. Slides were treated with Dako faramount aqueous mounting medium ready to use and then was applied to the coverslip. 


\section{Image and statistical analysis}

Cells were observed in an Olympus BX51 photomicroscope equipped with a KY-F58 CCD camera (JVC). The images were analyze and stored using the software Image-ProPlus on a personal computer. Ten cells were imaged and 1935 LDs were detected. For each LD area, perimeter, Maximum Feret's Diameter (MFD) and IOD were measured. The Feret's diameter (FD) is a measure of an object size along a specified direction, defined as the distance between the two parallel planes (or lines in case of planar images) restricting the object perpendicular to that direction. FD is generally used in optical microcopy to measure the size of irregularly shaped particles. ${ }^{13}$ Here we measured the MFD, the longest distance between any two points of the object, also known as maximum caliper diameter. The LDs' properties were measured using a semi-automatic procedure based on the automatic routines implemented in Image J with a strict control of an operator. The procedure and the parameters used to guide the routines were previously carefully checked and the results were compared with the ones obtained completely manually from an operator on a subset of LDs ( 200). With the help of contrast variation and color changing the operator was able to separate almost all the clusters of LDs in single LDs. The non resolved clusters were removed from the analysis, they were very few with respect the total amount of LDs (less than $3.3 \%)$. The statistical analysis was conducted with both Matlab R2007a (Mathworks) and EasyFit 5.5 (Mathwave).

\section{Results}

\section{Size distribution}

The images show that LDs are well stained and the image contrast is good (Figure 1a). This result is useful for the semi-automatic analysis procedure. The form of the 1935 LDs detected results almost perfect circular. Plotting area versus perimeter (Figure 1b) this visual impression is confirmed by the distribution of the experimental data in comparison with the perfect circularity represented by the red line.

The perfect circularity is verified when Area $=4 \pi \times[\text { Perimeter }]^{2}$. So the experimental data were fitted with a quadratic curve and the fit (dotted line) is very good ( $\mathrm{R} 2=0.999)$.

Figure 1c shows the histogram of the MFD of the LDs. LDs have MFD in the range 0.3-3.6 $\mu \mathrm{m}$. The histogram is clearly asymmetric and shows a long tail in the positive axis reflecting the presence of LDs with sizes very higher with respect the mean of the population. To avoid the dependence of the histogram representation to the choice of the sub-intervals or bins which cover the range of the data, the kernel density of the MFD distribution was calculated and it is visible in Figure 1d. Most of the LDs has a MFD around $0.5 \mu \mathrm{m}$. The more detailed representation of the data suggests a local secondary peak around $0.8 \mu \mathrm{m}$.

In order to analyze the distribution of the MFD of the LDs we tested the goodness of different distributions. In the widespread of asymmetric distributions we found that the experimental data can be fitted with a subset of distribution, most of them described with more than three parameters. In order to simplify the model we focused on distributions with no more than three parameters; in particular we found that the data can be fitted with the Generalized Extreme Value (GEV), lognormal and gamma functions. The GEV distribution is a family of continuous probability distributions developed within extreme value theory to combine the Gumbel, Fréchet and Weibull families also known as type I, II and III extreme value distributions (defined by three parameters: local parameter $\mu$, scale parameter $\sigma$, and shape parameter $\mathrm{k}$ ). The lognormal distribution is a continuous probability distribution of a random variable whose logarithm is normally distributed (defined by two parameters:
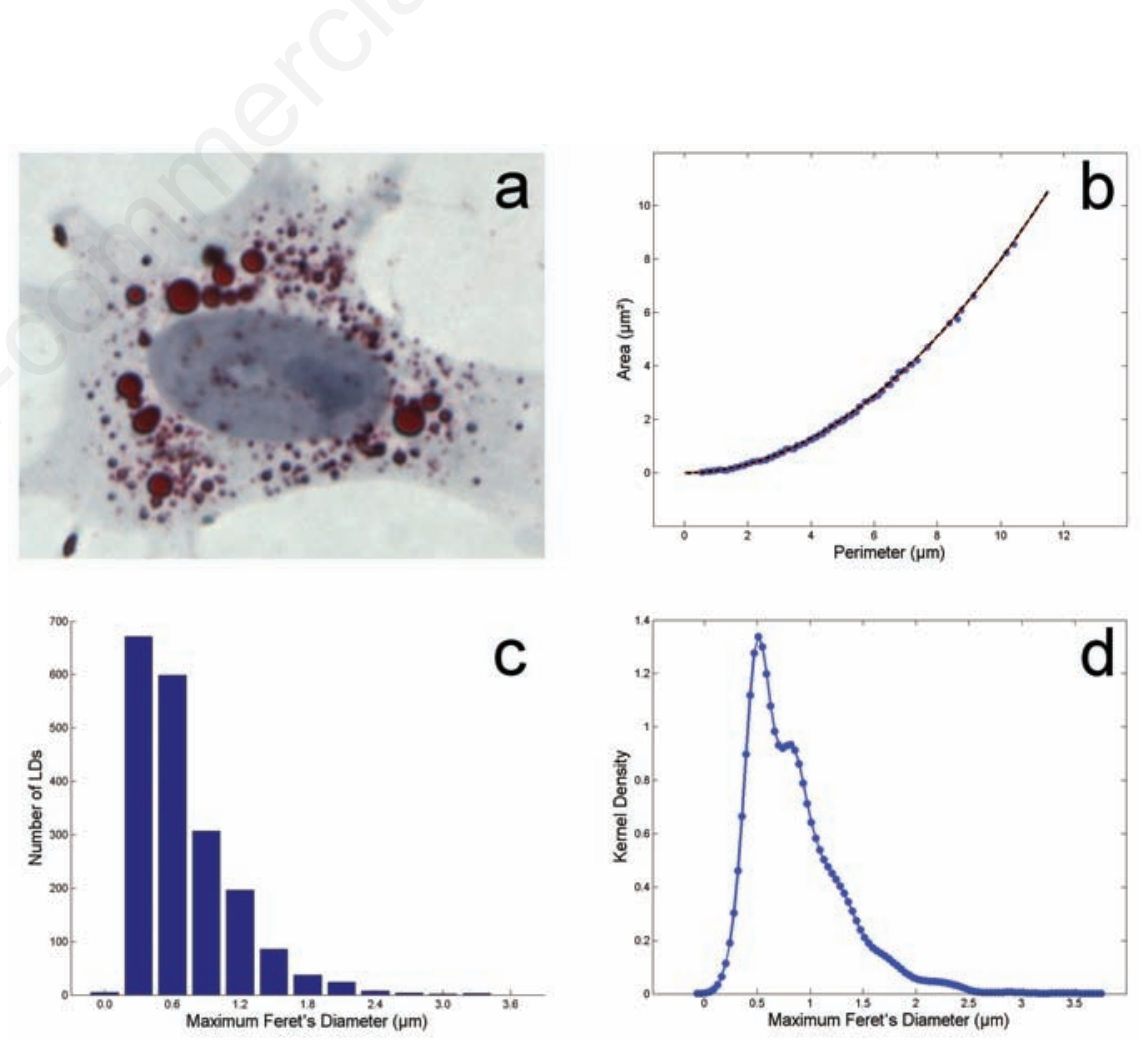

Figure 1. Oil Red $\mathrm{O}$ stained lipid droplets are well visible in the peri-nuclear region of a 3T3-L1 cell differentiated into adipocyte (a). The circularity of the lipid droplets is evident in the plot area vs perimeter (b); the red line represents the perfect circularity and the dotted line the best fit. Histogram (c) and kernel distribution (d) of the maximum Feret's diameter of the lipid droplets. the shape parameter $\sigma$ and the log-scale parameter $\mu$ ). The gamma distribution is a two-parameter family of continuous probability distributions with different parameterizations: we used here the shape parameter $\alpha$ and the rate parameter $\beta$. We obtained the subsequent fitting values: $\mathrm{k}=0.15731, \sigma=0.27612$ and $\mu=0.64964$ for GEV, $\sigma=0.46083$ and $\mu=-0.26163$ for lognormal and $\alpha=3.9329$ and $\beta=0.21852$ for gamma distribution. The goodness of the fitting was evaluated with tree different methods: GEV distribution obtained the fit with statistical test Kolmogorov Smirnov (KS) $=0.16886$ and rank 34, Anderson Darling (AD) $=33.3222$ and rank $20, \chi^{2}=1809.3$ and rank 43. Lognormal distribution obtained: statistical test $\mathrm{K}-\mathrm{S}=0.16661$ and rank $31, \mathrm{~A}-\mathrm{D}=32.865$ and rank $18, \chi^{2}=1714.5$ and rank 37 . Gamma distribution obtained: statistical test K-S $=0.13633$ and rank 2, A-D=35.777 and rank 25, $\chi^{2}$ $=382.59$ and rank 1. Despite all the three distributions can fit the data the gamma distribution obtained the best fit (lower rank values). The experimental data and the three distributions are visible in the same plot in Figure 2. Despite GEV distribution fits the peak of the experimental data distribution better than the others distributions, the gamma distribution is the best fitting curve considering the entire set of data. 


\section{Optical density distribution}

Figure 3a shows the histogram of the IOD of the LDs. Most of the LDs has low IOD, instead a small number presents a higher IOD as reflected in the asymmetric distribution. The kernel distribution is visible in Figure 2b. The values span from 2 to 410 . In order to fit the experimental data distribution we tried with the asymmetric curves described above: GEV, lognormal and gamma distributions. We obtained the subsequent fitting values: $\mathrm{k}=0.27225, \sigma=15.93$ and $\mu=25.809$ for $\mathrm{GEV}$; $\sigma=0.6653$ and $\mu=3.4766$ for lognormal; $\alpha=1.5249$ and $\beta=26.755$ for gamma distribution. Also for IOD the goodness of the fitting was evaluated with tree different methods. GEV distribution obtained the fit with statistical test $\mathrm{K}-\mathrm{S}=0.03519$ and rank $18, \mathrm{~A}-\mathrm{D}=2.7728$ and rank $15, \chi^{2}=56.292$ and rank 18 . Lognormal distribution obtained: statistical test $\mathrm{K}-\mathrm{S}=0.03207$ and rank $13, \mathrm{~A}-\mathrm{D}=2.4218$ and rank $11, \chi^{2}=48.446$ and rank 15 . Gamma distribution obtained: statistical test $\mathrm{K}$ $\mathrm{S}=0.12422$ and rank $30, \mathrm{~A}-\mathrm{D}=42.76$ and rank 27, $\chi^{2}=299.27$ and rank 29. Despite all the three distributions can fit the data only the lognormal distribution obtained the best fit. The three distributions used for the fit and the experimental data are visible in Figure 3c.

\section{Discussion}

In recent years ultrastructural analysis revealed the morphological polymorphism of

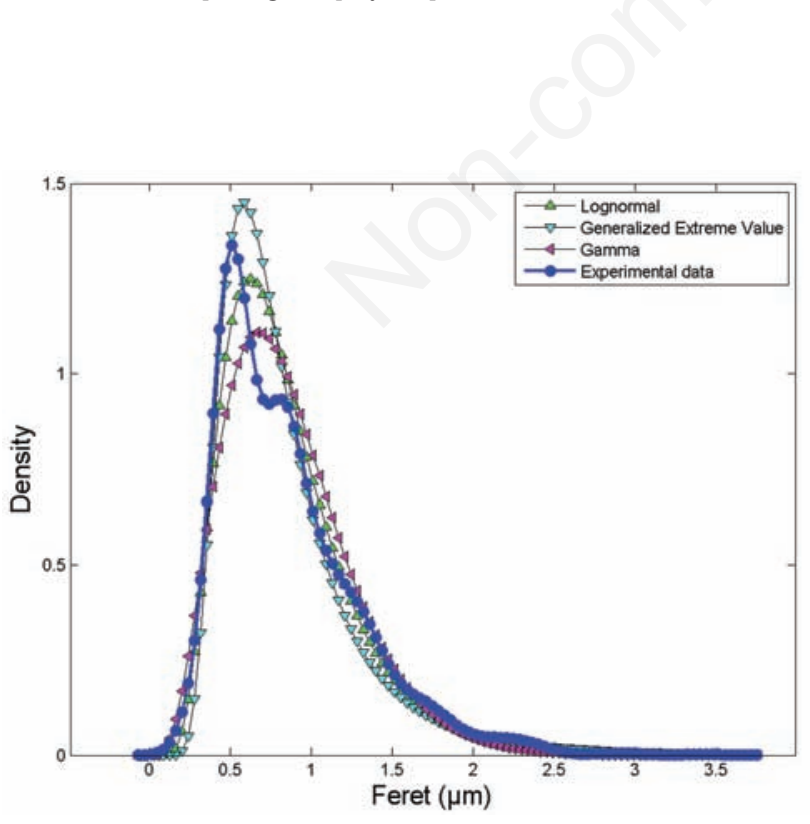

Figure 2. Kernel density of the maximum Feret's size of the lipid droplets and three fitting curves: Generalized Extreme Value, lognormal and gamma distributions. Despite Generalized Extreme Value distribution well fits the peak of the distribution, gamma distribution represents the best fit with respect the entire dataset. the adipose tissue with different lipid depots. $^{14,15}$ This results encourages the study of lipid storage in adipocytes. This work demonstrates that 3T3-L1 cells represent an excellent model to study the process of lipid accumulation in cytoplasm. The shape of the detected LDs resulted almost perfectly circular suggesting a homogeneous distribution of the transport through the surface of the LDs.
The planar images of the differentiated 3T3L1 cells showed some overlaps between the LDs. Many of them can be resolved using different thresholds and varying the contrast parameters in the representation of the images. The non-resolved clusters of LDs are very few and can be neglected when using a great number of LDs. In the planar images it is possible that small LDs located below the
Figure 3. Histogram (a) and kernel distribution (b) of integrated optical density of the lipid droplets (LDs). Comparison between kernel density of the integrated optical density data and three fitting curves: Generalized Extreme Value, lognormal and gamma distributions (c). The lognormal distribution represents the best fit of the data.
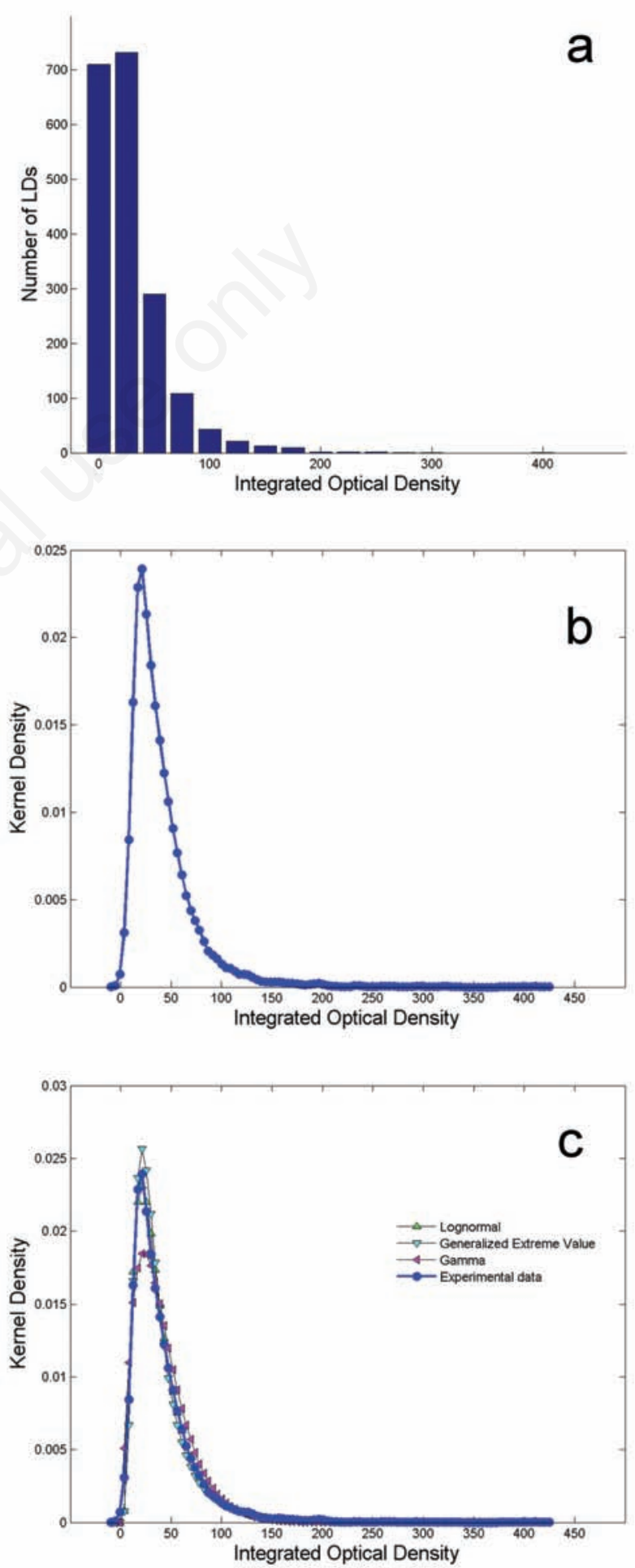
largest LDs along the $\mathrm{z}$-axis (respect the cells layered in the xy plane) were not observed. A new interesting approach could derive from the use of the confocal microscopy to analyze 3D images to extract a more detailed size distribution of the LDs. We described here the MFD and IOD distributions of the LDs in 5-days differentiated 3T3-L1 cells. The continuous distribution of the IOD shows the absence of eventual subpopulations of LDs characterized by different amounts of lipids. Ramirez-Zacarias and coworkers found that Oil red 0 specifically stains triglycerides and cholesteryl oletate ,but no other lipids and showed a linear relationship between the degree of staining and the lipid contents in fat cells. ${ }^{9}$ In case of 3T3 adipocytes lipid accumulation is mainly due to increase of triglyceride synthesis, but cholesterol or its derivatives did not seem to accumulate during adipocyte differentiation. ${ }^{16}$ Thus, it is possible to argue that the IOD curve presented here reflects also the continuous distribution in the triglycerides content in the LDs. The IOD data represent a template in order to test the effectiveness of different treatments. In particular variations in the IOD data distribution could be expected when the cells increase the synthesis of the triglycerides or the concentration of the lipids in the LDs. Comparing the IOD distribution before and after treatment it could be possible to acquire information on the lipids concentration in the new born LDs.

Many different pathologies involved in fat accumulation are related to changing in LDs number and size. Some studies analyzed the size of the LDs and the changing in the size after different treatments but they focused on the mean value and the standard deviation ${ }^{17}$ or on a subset of LDs randomly selected. ${ }^{18}$ In this study we measured all the detectable LDs in the analyzed cells and we propose a mathematical description of the entire size distribution of the LDS in order to create a standard population for future comparisons in different (healthy and pathological) conditions. The description of the entire size distribution can overcome the limitation due to the comparison between only the mean values and, for example, it can show differences in distributions with the same mean values. In general the curve representing a distribution contains many information not well represented by a single value. In this study we used curves characterized by two or three parameters which are related to the mean, the variance, the skewness and other properties of the distribution itself and a description with more than a single parameter could highlight changes, for instance, in the tail of the distributions.

We found that the best distribution to fit the distribution of the size of the LDs is a gamma curve. The local maximum in the MFD distribution around $0.8 \mu \mathrm{m}$ represents a problem for the fitting. We hypothesized that it could derive from the quantization of the measurements done on the computer screen. Using different magnifications of the images at the monitor on a sub-population of LDs ( 200) we did not observe the peak. Otherwise, the local maximum could be due to non homogeneity of the analyzed cells; in particular differences in LDs' size could reflect different ages of the cells themselves because it was observed that the size of the LDs can change along time. ${ }^{19}$ Another hypothesis is that the entire distribution could be considered the sum of two different distributions; in this case the increase in size of the LDs could occur in two or more steps: synthesis of triglycerides and formation of the small LDs (first step) and than fusion of the LDs (second step). The asymmetry of the kernel density distribution of the MFD of the LDs could be related with the increasing of the sizes of the LDs along time due to the fusion of the LDs themselves. Fusion of LDs was effectively observed by different authors with confocal microscopy. ${ }^{19,20}$ We found the best distribution for MFD and IOD of the LDs, but at the present time more than a single model could be valid to describe the LDs in 3T3-L1 5-days differentiated adipocytes. A mathematical description or more information regarding the birth of the LDs could help in finding the best mathematical model. In any case, we would like to propose a mathematical approach to quantify the modifications in order to evaluate many different pathologies or the growth of the LDs along time.

\section{References}

1. Barness LA, Opitz JM, Gilbert-Barness E. Obesity: genetic, molecular, and environmental aspects. Am J Med Genet A 2007;143A: 3016-34.

2. Mokdad AH, Marks JS, Stroup DF, Gerberding JL. Actual causes of death in the United States, 2000. JAMA 2004;291:1238-45.

3. Allison DB, Fontaine KR, Manson JE, Stevens J, VanItallie TB. Annual deaths attributable to obesity in the United States. JAMA 1999;282: 1530-8.

4. Haslam DW, James WP. Obesity. Lancet 2005;366:1197-209.

5. Grundy SM. Obesity, metabolic syndrome, and cardiovascular disease. J Clin Endocrinol Metab 2004;8:2595-600.

6. Cowherd RM, Lyle RE, McGehee RE Jr. Molecular regulation of adipocyte differentiation. Semin Cell Dev Biol 1999;10:3-10.

7. Li P, Carter G, Romero J, Gower KM, Watson J, Patel NA, et al. Clk/STY (cdc2like kinase 1) and Akt regulate alternative splicing and adipogenesis in 3T3-L1 preadipocytes. PLoS One 2013;8:e53268.

8. Greenberg AS, Coleman RA, Kraemer FB, McManaman JL, Obin MS, Puri V, et al. The role of lipid droplets in metabolic disease in rodents and humans. J Clin Invest 2011;121: 2102-10.

9. Ramírez-Zacarías JL, Castro-Mufiozledo F, Kuri-Harcuch W. Quantitation of adipose conversion and triglycerides by staining intracytoplasmic lipids with oil red 0 . Histochemistry 1992;97:493-7.

10. Porzionato A, Sfriso MM, Macchi V, Rambaldo A, Lago G, Lancerotto L, et al. Decellularized omentum as novel biologic scaffold for reconstructive surgery and regenerative medicine. Eur J Histochem 2013;57:e4.

11. Goodpaster BH, Theriault R, Watkins SC, Kelley DE. Intramuscular lipid content is increased in obesity and decreased by weight loss. Metabolism 2000;49:467-72.

12. Ducharme NA, Bickel PE. Lipid droplets in lipogenesis and lipolysis. Endocrinology 2008;149:942-9.

13. Walton WH, Feret's statistical diameter as a measure of particle size. Nature 1948; 162:329-30.

14. Sbarbati A, Accorsi D, Benati D, Marchetti L, Orsini G, Rigotti G, et al. Subcutaneous adipose tissue. Eur J Histochem 2010; 54:e48.

15. Panettiere P, Accorsi D, Marchetti L, Minicozzi AM, Orsini G, Bernardi P et al. The trochanteric fat pad. Eur J Histochem 2011; 55:e16.

16. Green H., Kehinde 0. Sublines of mouse 3T3 cells that accumulate lipid. Cell 1974; 1:113-6.

17. Blouin CM, Le Lay S, Eberl A, Köfeler HC, Guerrera IC, Klein C, Le Liepvre X, et al. Lipid droplet analysis in caveolin-deficient adipo-cytes: alterations in surface phospholipid composition and maturation defects. J Lipid Res 2010;51:945-56.

18. Stringer DM, Zahradka P, Declercq VC, Ryz NR, Diakiw R, Burr LL, et al. Modulation of lipid droplet size and lipid droplet proteins by trans-10,cis-12 conjugated linoleic acid parallels improvements in hepatic steatosis in obese, insulin-resistant rats. Biochim Biophys Acta 2010;1801:1375-85.

19. Boström P, Rutberg M, Ericsson J, Holmdahl P, Andersson L, Frohman MA, et al. Cytosolic lipid droplets increase in size by microtubule-dependent complex formation. Arterioscler Thromb Vasc Biol 2005; 25:1945-51.

20. Murphy S, Martin S, Parton RG. Quantitative analysis of lipid droplet fusion: inefficient steady state fusion but rapid stimulation by chemical fusogens. PLoS One 2010;5:e15030. 